\title{
EXISTENCE THEOREMS OF OPTIMAL CONTROL PROBLEMS IN BANACH SPACES*
}

\author{
Hou, SHui-Hung ${ }^{\dagger}$ \\ Department of Mathematics and Department of Computer Information and Control Engineering, University of \\ Michigan, Ann Arbor, Michigan, U.S.A.
}

(Received 2 November 1981)

Key words and phrases: Optimal control, maximal monotone, property (Q), multifunction, Carathéodory mapping.

\section{INTRODUCTION}

THE SUBJECT of optimization problems in the theory of distributed parameter control systems has gained much interest. This is readily seen in the survey on the subject by Robinson [20] and Russell [21]. Linear systems governed by partial differential equations have been given the most attention, cf. Axelband [2], Aziz et al. [3], Balakrishnan [4] and Lions [18], to name a few.

In the present paper we first seek sufficient conditions for the cxistencc of admissible pairs $(x, u)$ of optimal control systems monitored by nonnecessarily linear operational equations of the form

$$
\begin{aligned}
& (E x)(t)+(\Lambda x)(t)=g(t, u(t)), \\
& u(t) \in \omega(t), \quad t \in[0, T],
\end{aligned}
$$

where the function $u$ is the control function, and $x$ defined on $[0, T]$ is the state or trajectory and both $u$ and $x$ have values in Banach spaces. Secondly we prove existence theorems for optimal solutions for Lagrange and Mayer problems for systems monitored by the above operational equations.

On the operators $E$ and $\Lambda$ and function $g$ we shall only assume that $E$ is a linear monotone operator. On the operator $\Lambda$ we assume a weak continuity condition and a mild coercivity condition on $\Lambda-g$.

The state and control functions in the operational equations are separated from each other, thus including linear systems as a special case. This separation feature enables us to prove the closure property of admissible trajectories and compactness for attainable sets, as seminormality conditions, for example property (Q) (cf. Cesari [8], Hou [12]), are readily satisfied.

Finally we study several examples in detail.

\section{STATEMENT OF THE CONTROL PROBLEM}

Let $H$ be a real Hilbert space with inner product $(,)_{H}$ and $V$ be a real separable reflexive Banach space. Denote by $V^{*}$ the dual of $V$ and assume that $V \subset H \subset V^{*}$ with each inclusion mapping continuous and densely defined. Let $\left\langle v^{*}, v\right\rangle$ or $\left\langle v, v^{*}\right\rangle$ denote the duality pairing

* This research was partially supported by AFOSR Research Project 77-3211 at the University of Michigan.

$\dagger$ Presently at the Department of Mathematical Studies, Hong Kong Polytechnic, Hong Kong. 
between $v^{*}$ in $V^{*}$ and $v$ in $V$; if $v, v^{*} \in H$, this is the inner product in $H$. Denote by $|\cdot|_{H}$ the norm in $H$ and by $\|\cdot\|$ and $\|\cdot\|_{*}$ the norms in $V$ and $V^{*}$ respectively.

Let $J \equiv[0, T], T>0$, be a compact interval of the real line $\mathbb{R}$. For any separable metric space $Z$ the symbol $\mathcal{M}(J, Z)$ denotes the set of all measurable functions on $J$ with values in $Z$.

For $1<p<\infty$, let $X \equiv L_{p}(J, V)$ be the space of all $V$-valued strongly measurable functions $x(t)$ defined a.e. in $J$ such that $\|x(t)\|^{p}$ is Lebesgue-integrable over $J$. It is well known that $X$ is a Banach space with the norm defined by

$$
\|x\|_{X} \equiv\left[\int_{J}\|x(t)\|^{p}\right]^{1 / p}
$$

Because of the reflexivity of $V$, the dual $X^{*}$ is isometrically isomorphic to $L_{q}\left(J, V^{*}\right)$ with $1 / p+1 / q=1$ (see Leonard [17] for a short proof). The correspondence is given as follows: for each $F \in X^{*}$, there exists a unique $g \in L_{q}\left(J, V^{*}\right)$ such that

$$
F(f)=\int_{J}\langle g(t), f(t)\rangle \mathrm{d} t
$$

for all $f$ in $X$ and

$$
\|F\|_{X^{*}}=\left[\int_{J}\|g(t)\|^{q} \mathrm{~d} t\right]^{1 / q}
$$

We will always identify the dual $X^{*}$ with $L_{q}\left(J, V^{*}\right)$ in this manner. Thus $X^{*}=L_{q}\left(J, V^{*}\right)$ and the duality pairing between $X$ and $X^{*}$ is given by

$$
\{f, g\} \equiv \int_{J}\langle g(t), f(t)\rangle \mathrm{d} t
$$

for any $f \in X$ and $g \in X^{*}$. Since $V^{*}$ is reflexive, we have $X^{* *}=\left(L_{q}\left(J, V^{*}\right)\right)^{*}=$ $L_{p}\left(J, V^{* *}\right)=L_{p}(J, V)=X$ and hence $X$ is reflexive.

Let $E, \Lambda$, be two operators with domains $D(E), D(\Lambda) \subseteq X$ and values in $X^{*}$. Let $U$ be a separable complete metric space and $\omega: J \rightarrow U$ be a measurable multifunction with closed values (cf. Hou[13]).

We shall be concerned with the control system described by

$$
\begin{array}{cc}
(E x)(t)+(\Lambda x)(t)=g(t, u(t)) \\
u(t) \in \omega(t) \quad \text { a.e. in } J .
\end{array}
$$

We shall hereafter refer this system by $(P)$.

We say a pair $(x, u)$ is admissible for this system provided $x \in D(E) \cap D(\Lambda), u \in M(J, U)$ and (1.1) and (1.2) are satisfied. We say that $x$ is an admissible trajectory or state and that $u$ is an admissible control.

Remark. In view of applications, the right-hand side of (1.1) is written as a function of control rather than as a control by itself. Systems with $g(t, x, u)$ will be considered in a later paper (cf. Hou [14]).

The function $g: J \times U \rightarrow V^{*}$ in (1.1) is assumed to be a Carathéodory function; more 
precisely, $g(t, u)$ is strongly measurable in $t$ for each fixed $u \in U$ and demicontinuous $\dagger$ in $u$ for almost every $t$ in $J$.

Under this assumption, $g(\cdot, u(\cdot))$ is then strongly measurable from $J$ to $V^{*}$ for any $u \in$ $M(J, U)$ (cf. appendix, theorem 3). Suppose further that $g(\cdot, u(\cdot))$ belongs to $X^{*}$, then we can define an operator $N_{u}: D(\Lambda) \rightarrow X^{*}$ by

$$
\left(N_{u} x\right)(t) \equiv(\Lambda x)(t)-g(t, u(t))
$$

Thus (1.1), (1.2) may be viewed as

$$
\begin{array}{lr}
E x+N_{u} x=0 & \text { in } X^{*}, \\
u(t) \in \omega(t) & \text { a.e. in } J .
\end{array}
$$

\section{BASIC ASSUMPTIONS AND THEOREMS CONCERNING THE EXISTENCE OF SOLUTIONS}

In this section, we study the problem of existence of admissible trajectories of $(\mathrm{P})$ corresponding to a given control $u$ with $u(t) \in \omega(t)$ a.e. in $J$.

To solve the problem just posed, we shall make the following basic assumptions:

(A1) $E: X \rightarrow X^{*}$ is a linear, maximal monotone operator with domain $D(E) \subset X$.

(A2) $\Lambda: X \rightarrow X^{*}$ is an operator with domain $D(\Lambda)=X$ and it is sequentially weakly continuous from $X$ to $X^{*}$; i.e., $\Lambda$ maps weakly convergent sequences of $X$ into sequences weak-star convergent in $X^{*}$.

(A3) For any measurable selection $u$ of $\omega, g(\cdot, u(\cdot)) \in X^{*}$.

(A4) There exists a bounded closed convex set $K \subset X$ containing the origin in its interior such that $\left\{N_{u} x, x\right\} \geqslant 0$ for any measurable selection $u$ of $\omega$ and for any $x \in \partial K$. $\partial K$ denotes the boundary of $K$ and $N_{u}$ is defined as in (1.3), (we consider this condition (A4) as a mild coercivity condition on $\Lambda-g$ ).

Before stating an existence theorem for the nonlinear problem (1.4), (1.5), we need the following property of $M$-type operators from a Banach space $B$ to its dual $B^{*}$.

Definition 2.1. A single-valued mapping $T$ of $D(T)=B$ into $B^{*}$ is called of type $M$ if it satisfies the following two conditions:

(M1) If $\left\{x_{i}\right\}$ is a net such that $\left\|x_{i}\right\|_{B} \leqslant C, x_{i} \rightarrow x$ in $B$,

$$
T x_{i} \stackrel{w^{*}}{\rightarrow} x^{*} \text { in } B^{*}
$$

and $\lim \sup \left\langle T x_{i}, x_{i}\right\rangle \leqslant\left\langle x^{*}, x\right\rangle$, then $T x=x^{*}$.

(M2) The restriction of $T$ to any finite-dimensional subspace of $B$ is continuous with respect to the weak-star topology of $B^{*}$.

Remark. The concept of $M$-type mapping was first introduced by Brézis [6].

We now state an existence theorem for nonlinear equations which is a special case of a theorem due to Kenmochi [16, theorem 2].

$\dagger$ A function $z: U \rightarrow V^{*}$ is said to be demicontinuous if $z\left(u_{n}\right)-z(u)$ for any sequence $\left\{u_{n}\right\}$ converging to $u$ in $U$. $\rightarrow$ denotes weak convergence in $V^{*}$. Note that the weak and weak-star topologies in $V^{*}$ coincide, since $V^{*}$ is reflexive. 
THEOREM 2.2. Let $K$ be a bounded closed convex subset of a reflexive Banach space $X$ containing the origin in its interior. Let $E$ be a linear, maximal monotone operator of $D(E) \subseteq X$ to $X^{*}$ and $N$ a bounded mapping of type $M$ from $D(N)=X$ to $X^{*}$. Suppose that $\{N x, x\} \geqslant 0$ for all $x \in \partial K$, where $\partial K$ is the boundary of $K$. Then the set $S \equiv\{x \in K: E x+$ $N x=0\}$ is nonempty.

Using theorem 2.2, we can obtain the following main existence theorem for $(\mathrm{P})$ :

THEOREM 2.3. Suppose that $E, \Lambda, g$, of (P) satisfy assumptions (A1)-(A4). Then for any measurable selection $u$ of $\omega$, there exists an admissible trajectory $x_{u} \in K$ for $(\mathrm{P})$. Moreover, the set

$$
\Phi \equiv\{x \in K:(x, u) \text { admissible pair for some } u\}
$$

is relatively sequentially weakly compact in $X \equiv L_{p}(J, V)$.

Proof. We must show that for any measurable selection $u$ of $\omega$, the equation $E x+N_{u} x$ $=0$ has a solution $x_{u}$ in $K$. In view of theorem 2.2. and the definition of $N_{u}$, it is enough to show that $\Lambda$ is a bounded mapping of type $M$.

For boundedness of $\Lambda$, we apply the lemma below.

LEMMA 2.4. Let $B_{1}$ be a reflexive Banach space and $B_{2}$ a Banach space. If $f$ maps weakly convergent sequences of $B_{1}$ into sequences weakly convergent in $B_{2}$, then $f$ is bounded.

Now by assumption (A2), $A$ is sequentially weakly continuous, whence $\Lambda$ is bounded by lemma 2.4 .

To see that $\Lambda$ is of type $M$, we need only to demonstrate that $\Lambda$ satisfies (M1), as (M2) is obviously fulfilled because of (A2). To this end, let $\left\{x_{i}\right\}$ be a net in $X$ such that $\left\|x_{i}\right\|_{X} \leqslant C$, $x_{i} \rightarrow x$ and $\Lambda x_{i} \rightarrow x^{*}$, we intend to show $\Lambda x=x^{*}$. Since $V$ is separable, the space $X \equiv L_{p}(J, V)$ is also separable (cf. Warga [23, theorem I.5.8]). The closed ball $\mathscr{B} \equiv\left\{z \in X:\|z\|_{X} \leqslant C\right\}$ is weakly compact because $X$ is reflexive. Therefore the weak topology of $\mathscr{B}$ is a metric topology (Dunford \& Schwartz [11, theorem V.6.3]). Thus there exists a subsequence $\left\{b_{n}\right\} \subset \mathscr{B}$ of $\left\{x_{i}\right\}$ such that $b_{n} \rightarrow x$. It follows from the sequential weak continuity of $\Lambda$ that $\Lambda b_{n} \rightarrow \Lambda x$. Hence $\Lambda x=x^{*}$, proving that $\Lambda$ is of type $M$.

All the hypotheses of theorem 2.2 are now satisfied, therefore for each measurable selection $u$ of $\omega$, there exists an admissible trajectory in $K$. Since the set $\Phi \subseteq K$ and $K$ is bounded, $\Phi$ is therefore relatively weakly compact, hence relatively sequentially weakly compact by the Eberlein-Smulian theorem (cf. Dunford \& Schwartz [11, theorem V.6.1]). This completes the proof of theorem 2.3.

It remains to prove Lemma 2.4 .

Proof of lemma 2.4. We argue by contradiction. Suppose there is a bounded sequence $\left\{b_{n}\right\}$ in $B_{1}$ such that $\left\|f\left(b_{n}\right)\right\|_{B_{2}} \rightarrow \infty$. By the reflexivity of $B_{1},\left\{b_{n}\right\}$ has a weakly convergent subsequence $\left\{b_{n_{j}}\right\}$. By hypothesis, $\left\{f\left(b_{n_{j}}\right)\right\}$ is weakly convergent and henec norm-bounded. But this contradicts the fact that $\left\|f\left(b_{n}\right)\right\|_{B_{2}} \rightarrow \infty$. Lemma 2.4 is thereby proved.

\section{PROPERTIES OF THE SOLUTIONS}

We need the following lemma due to Brézis [5] for the next result. 
Lemma 3.1. Let $X$ be a reflexive Banach space and let $D(\mathscr{A})$ be a linear subspace of $X$ and $A$ be a linear, monotone, single valued operator from $D(\mathscr{A})$ to $X^{*}$. Then the following assertions are equivalent.

(1) $\mathscr{A}$ is maximal monotone.

(2) $\mathscr{A}$ is a densely defined, closed linear operator such that its adjoint $\mathscr{A}^{*}$ is monotone.

(3) $A$ is a densely defined, closed, linear operator such that $\mathbb{A}^{*}$ is maximal monotone.

In order to prove the set $\Phi$, defined in theorem 2.3 , is closed under sequential convergence, we impose two more assumptions on the mapping $g$ of (1.1).

(B1) There exists an $l \in L_{q}(J, \mathbb{R})$ such that $\|g(t, u(t))\|^{*} \leqslant l(t)$ a.e. for any measurable selection $u$ of $\omega$.

(B2) For almost every $t$ in $J$, the set $g(t, \omega(t))$ is closed and convex in $V^{*}$.

Remark. We note that assumption (B1) implies (A3).

THEOREM 3.2. Let the assumptions (A1)-(A4), (B1) and (B2) hold. Then the set $\Phi$ is sequentially weakly compact in $X$. Moreover, $\Phi$ is sequentially weakly compact in the space $D(E)$ endowed with the topology induced by the graph norm associated with $E$.

Remark. The graph norm is defined by

$$
\|x\|=\|x\|_{X}+\|E x\|_{X^{*}}
$$

Since $E$ is linear maximal monotone, it is densely defined, closed and linear from $X$ to $X^{*}$ by lemma 3.1. Hence $D(E)$ is a Banach space under the graph norm. Moreover $D(E)$ is continuously imbedded into $X$ and dense in $X$ and it is reflexive.

Proof of theorem 3.2. Let $\left\{x_{n}\right\}$ be a sequence in $\Phi$ and suppose $x_{n} \rightarrow x$. Then $x \in K$. We must show $x \in \Phi$. Since $x_{n} \in \Phi$, there exists measurable selections $u_{n}$ of $\omega$ such that, for a.a $t$ in $J,\left(E x_{n}\right)(t)+\left(\Lambda x_{n}\right)(t)=g\left(t, u_{n}(t)\right)$. By (B1) we get $\left\|\left(E x_{n}\right)(t)+\left(\Lambda x_{n}\right)(t)\right\|_{*} \leqslant l(t)$ a.e. This

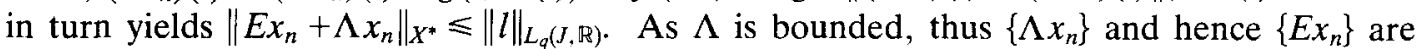
bounded. We may assume, by relabelling if necessary, that $\Lambda x_{n} \rightarrow \xi$ and $E x_{n} \rightarrow \eta$ for some $\xi, \eta \in X^{*}$. The fact that $E$ is closed by lemma 3.1 implies $E x=\eta$. Also by (A2), we have $\Lambda x=\xi$. Thus $E x_{n}+\Lambda x_{n} \rightarrow E x+\Lambda x$.

Let us define $\mathscr{G}(t, x) \equiv g(t, \omega(t))$ for every $(t, x) \in J \times X$. Obviously $\mathscr{G}$ is a multifunction from $J \times X_{w^{\dagger}}$ to $V^{*}$ satisfying property (Q) with respect to $x$ for all $t \in J$. As $x_{n} \rightarrow x$, $E x_{n}+\Lambda x_{n} \rightarrow E x+\Lambda x$, and $\left(E x_{n}\right)(t)+\left(\Lambda x_{n}\right)(t) \in g(t, \omega(t))=\mathscr{G}\left(t, x_{n}\right)$ a.e. in $J$, we have by Cesari's closure theorem (cf. Appendix, theorem 1) that

$$
(E x)(t)+(\Lambda x)(t) \in \mathscr{G}(t, x)=g(t, \omega(t)) \quad \text { a.e. }
$$

To see that $x$ is an admissible trajectory, we must produce a measurable selection $u$ of $\omega$ such that $(E x)(t)+(\Lambda x)(t)=g(t, u(t))$ a.e. Since $V$ is separable and reflexive, there exists a 
countable family $\mathscr{Y} \subset V^{* *}$ that separates points of $V^{*}$ (cf. Hille \& Phillips [15, theorem 2.8.5]). The map $g$ is Carathéodory and $\omega$ is measurable with closed values. Thus we can apply the implicit function theorem in the Appendix to produce a measurable selection $u$ of $\omega$ as required. Therefore $(x, u)$ is an admissible pair and whence $x \in \Phi$. This completes the proof.

\section{THEOREMS CONCERNING THE EXISTENCE OF OPTIMAL SOLUTONS FOR MAYER AND LAGRANGE TYPE PROBLEMS}

In this section we solve two specific optimization problems making use of the results of sections 2 and 3.

The first problem we consider is of Mayer type, i.e. minimizing a cost functional $I[x]$ over the class $\Phi$ of admissible trajectories $x$.

THEOREM 4.1. Suppose the hypotheses of theorem 3.2 are satisfied. Let $I[x]$ be a weakly lower semicontinuous functional on $X$ which is bounded from below on every bounded subset of $X$. Then the functional $I[x]$ has an absolute minimum in $\Phi$.

Proof. Since $I$ is bounded below on $\Phi$, the infimum $i$ of $I$ on $\Phi$ is finite, and we take a sequence $\left\{x_{k}\right\} \subset \Phi$ with $I\left[x_{k}\right] \rightarrow i$ as $k \rightarrow \infty$. The compactness of $\Phi$ (theorem 3.2) implies that there is a subsequence, again denoted by $\left\{x_{k}\right\}$, such that $x_{k} \rightarrow x \in \Phi$ with $I[x] \geqslant i$. By the lower semicontinuity of $I$, we have $I[x] \leqslant i$ and hence $I[x]=i$. This completes the proof of theorem 4.1.

We need the following compactness lemmas due to Serierstad [22].

Lemma 4.2. Let $U$ be a separable Banach space and $G$ be a finite measure space. Let $A$ be a closed convex set in $U$ with the property that bounded subsets of $A$ are relatively weakly compact. Let $S$ be a norm bounded subset of the space $L_{r}(G, U)$ made up of functions whose values are in $A \subset U$. Then $S$ is relatively weakly compact for $1<r<\infty$.

THEOREM 4.3. Suppose that the hypotheses of theorem 2.3 are satisfied and $g(t, u) \equiv B(t) u$, where $\{B(t) ; t \in J\}$ is a family of bounded linear maps from $U$ to $V^{*}$. Moreover the space $U$ is assumed to be a separable Banach space and $\omega: J \rightarrow U$ is a measurable multifunction with closed convex values; each $\omega(t)$ being a subset of a closed convex set $A$ in $U$ with the property that bounded subsets of $A$ are relatively compact and there exists an integrable function $h \in L_{r}(J, \mathbb{R}), 1<r<\infty$, such that $\sup \left\{\|v\|_{U}: v \in \omega(t)\right\} \leqslant h(t)$ a.e. in $J$. Let $I[x, u]$ be a weakly lower semicontinuous functional which is bounded below on the class of admissible pairs

$$
\Omega \equiv \Phi \times\left\{u:\|u\|_{L_{r}(J, U)} \leqslant\|h\|_{L_{r}(J, \mathrm{R})} \text { and } u(t) \in \omega(t) \text { a.e. }\right\} .
$$

Then $I[x, u]$ has an absolute minimum in $\Omega$.

Proof. Since $I$ is bounded below on $\Omega$, the infimum $i$ of $I$ on $\Omega$ is finite, and we take a sequence of admissible pairs $\left(x_{k}, u_{k}\right) \in \Omega, k=1,2, \ldots$, with $I\left[x_{k}, u_{k}\right] \rightarrow i$ as $k \rightarrow \infty$. Then we may assume by theorem 2.3 and lemma 4.2 that there is a subsequence, again denoted by $\left(x_{k}, u_{k}\right)$, such that $x_{k} \rightarrow x$ and $u_{k} \rightarrow u$. By the weak continuity of $E$ and $\Lambda$, we have $E x_{k}+\Lambda x_{k} \rightarrow E x+\Lambda x$. Since the $B(t)$ are linear, the operator $B: L_{r}(J, U) \rightarrow X^{*}$, defined by $(B u)(t) \equiv B(t) u(t)$, is also lincar and we derive $B u_{k} \rightarrow B u$. Thus $E x+\Lambda x=B u$. The sets 
$\omega(t)$ are closed and convex by hypothesis. Since $u_{k} \rightarrow u$ and $u_{k}(t) \in \omega(t)$ a.e., we conclude that $u(t) \in \omega(t)$ a.e. by force of Cesari's closure theorem. Thus $(x, u) \in \Omega$ and $I[x, u] \geqslant i$. By the lower semicontinuity property of $I$, we have $I[x, u] \leqslant i$. Hence $I[x, u]=i$. This completes the proof.

Remark. The assertion of theorem 4.3 remains valid when $U$ is reflexive; it is also true for $r=1$ if each $\omega(t)$ is a subset of a weakly compact set $\mathscr{C}$ of $U$ in view of the next lemma due to Diestel [10].

LEMMA 4.4. Let $\mathscr{G}$ be a weakly compact convex subset of a Banach space $U$ and consider the set

$$
\tilde{\mathscr{C}} \equiv\left\{f \in L_{1}(J, U): f(t) \in \mathscr{C} \text { a.e. in } J\right\}
$$

Then $\overline{\mathscr{C}}$ is weakly compact in $L_{1}(J, U)$.

We now turn to the second optimization problem which is of Lagrange typc; i.c. minimizing a cost functional of the form

$$
I[x, u] \equiv \int_{J} f_{0}(t,(M x)(t), u(t)) \mathrm{d} t
$$

over the class $\Omega \equiv\{(x, u): x \in \Phi\}$ of admissible pairs.

We shall make use of the following assumptions.

(L1) Let $Y$ be a Banach space and $f_{0}$ a Carathéodory map from $J \times Y \times U \rightarrow \mathbb{R}$; i.e., $f_{0}(\cdot, y, u)$ is measurable for fixed $(y, u)$ and $f_{0}(t, \cdot, \cdot)$ is continuous for a.e. $t \in J$.

(L2) $M: D(E) \rightarrow L_{p}(J, Y)$ maps weakly convergent sequences in $D(E)$ into sequences strongly convergent in $L_{p}(J, Y)$.

(L3) There are $b \in V$ and $\psi \in L_{1}(J, \mathbb{R})$ with $\psi(t) \geqslant 0$ such that

$$
f_{0}(t, y, u) \geqslant\langle b, g(t, u)\rangle-\psi(t)
$$

for all $(t, y, u)$ in $J \times Y \times U$.

(L4) The multifunction 2: $J \times Y \rightarrow \mathbb{R} \times \mathrm{V}^{*}$ defined by

$$
2(t, y) \equiv\left\{\left(z^{0}, z\right): z^{0} \geqslant f_{0}(t, y, u), z=g(t, u) \text { and } u \in \omega(t) \text { a.e. }\right\}
$$

has property $(Q)$ with respect to $y$ for almost every $t$.

(L5) $I[x, u]$ is infinite for some $(x, u)$ in $\Omega$.

THEOREM 4.5. Let the assumptions (L1)-(L5) hold. Suppose the hypotheses of the theorem 2.3 are satisfied. Then the functional $I[x, u]$ defined by (4.1) has an absolute minimum in $\Omega$.

Proof. Let $r \equiv \inf I[x, u]<+\infty$. Choose a sequence $\left(x_{k}, u_{k}\right)$ in $\Omega$ such that $\lim I\left[x_{k}, u_{k}\right]=$ $r$. We first show $r>-\infty$. By the relative compactness of $\Phi$ (theorem 2.3) we may assume $x_{k} \rightarrow x$. Since $E, \Lambda$ are sequentially weak continuous, $E x_{k}+\Lambda x_{k} \rightarrow E x+\Lambda x$. From (L3) we 
get, for a.e. $t$,

$$
\begin{aligned}
f_{0}\left(t,\left(M x_{k}\right)(t), u_{k}(t)\right) & \geqslant-\psi(t)+\left\langle b, g\left(t, u_{k}(t)\right)\right\rangle \\
& =-\psi(t)+\left\langle b,\left(E x_{k}\right)(t)+\left(\Lambda x_{k}\right)(t)\right\rangle .
\end{aligned}
$$

Since $E x_{k}+\Lambda x_{k} \rightarrow E x+\Lambda x,\left\{E x_{k}+\Lambda x_{k}\right\}$ is therefore bounded and hence the integral

$$
\int_{\mathrm{J}}\left\langle b,\left(E x_{k}\right)(t)+\left(\Lambda x_{k}\right)(t)>\mathrm{d} t\right.
$$

is necessarily bounded. An integration of (4.2) shows that $I\left[x_{k}, u_{k}\right]$ is bounded below. Hence $r>-\infty$.

By (L2) we may assume, by choice of subsequence if necessary, that $\left(M x_{k}\right)(t) \rightarrow(M x)(t)$ a.e. in $J$. As $E x_{k}+\Lambda x_{k} \rightarrow E x+\Lambda x$, the Banach-Saks-Mazur theorem (cf. Dunford \& Schwartz [11]) enables us to construct a sequence of finite convex combinations $\left\{h_{n}\right\}$ such that

$$
h_{n}(t)=\sum_{i} \lambda_{i n}\left[\left(E x_{n+i}\right)(t)+\left(\Lambda x_{n+i}\right)(t)\right]
$$

with

$$
\sum_{i} \lambda_{i n}=1, \lambda_{i n} \geqslant 0, \text { and } h_{n}(t) \rightarrow(E x)(t)+(\Lambda x)(t)
$$

strongly a.e. Let us define a sequence

$$
v_{n}(t) \equiv \sum_{i} \lambda_{i n} f_{0}\left(t,\left(M x_{n+i}\right)(t), u_{n+i}(t)\right)
$$

where $\lambda_{\text {in }}$ are as in (4.3).

Let $v(t) \equiv \lim \inf v_{n}(t)$. From (4.3), (4.4) and (L3) we get

$$
\begin{aligned}
v_{n}(t) & -\left\langle b, h_{n}(t)\right\rangle \\
& =\sum_{i} \lambda_{i n}\left[f_{0}\left(t,\left(M x_{n+i}\right)(t), u_{n+i}(t)\right)-\left\langle b,\left(E x_{n+i}\right)(t)+\left(\Lambda x_{n+i}\right)(t)\right\rangle\right] \\
& =\sum_{i} \lambda_{i n}\left[f_{0}\left(t,\left(M x_{n+i}\right)(t), u_{n+i}(t)\right)-\left\langle b, g\left(t, u_{n+i}(t)\right)\right\rangle\right] \\
& \geqslant \sum_{i} \lambda_{i n}(-\psi(t))=-\psi(t) .
\end{aligned}
$$

Hence we may apply Fatou's lemma to obtain

$$
\begin{aligned}
\lim & \inf \int_{J}\left[v_{n}(t)-\left\langle b, h_{n}(t)\right\rangle\right] \mathrm{d} t \\
\geqslant & \int_{J} \lim \inf \left[v_{n}(t)-\left\langle b, h_{n}(t)\right\rangle\right] \mathrm{d} t \\
= & \int_{J}[v(t)-\langle b,(E x)(t)+(\Lambda x)(t)\rangle] \mathrm{d} t
\end{aligned}
$$

and

$$
v(t) \quad\langle b,(E x)(t)+(\Lambda x)(t)\rangle \geqslant-\psi(t) \text { a.e. }
$$


From (4.5) we obtain

$$
\int_{J} v(t) \mathrm{d} t \leqslant \liminf \int_{J} v_{n}(t) \mathrm{d} t
$$

But from (4.4) we have

$$
\begin{gathered}
\lim \inf \int_{J} v_{n}(t) \mathrm{d} t=\liminf \sum_{i} \lambda_{i n} \int_{J} f_{0}\left(t,\left(M x_{n+i}\right)(t), u_{n+i}(t)\right) \mathrm{d} t \\
=\lim \inf \sum_{i} \lambda_{i n} I\left[x_{n+i}, u_{n+i}\right]=r .
\end{gathered}
$$

Thus from this result with (4.6) and (4.7), we see that $v$ is integrable and hence

$$
\int_{J} v(t) \mathrm{d} t \leqslant r
$$

We next show that $(v(t),(E x)(t)+(\Lambda x)(t)) \in 2(t,(M x)(t))$ a.e. Let us fix a $t$ in $J$ at which the set-valued map $2(t, y)$ has property (Q) with respect to $y$, and for which $\left(M x_{n}\right)(t) \rightarrow$ $(M x)(t), v_{n}(t) \rightarrow v(t)$, and $h_{n}(t) \rightarrow(E x)(t)+(\Lambda x)(t)$ by choices of appropriate sub-sequences if necessary. Then for each $n$, we have

$$
\begin{aligned}
& \left(v_{n}(t), h_{n}(t)\right)=\sum_{i} \lambda_{i n}\left[f_{0}\left(t,\left(M x_{n+i}\right)(t), u_{n+i}(t)\right),\left(E x_{n+i}\right)(t)+\left(\Lambda x_{n+i}\right)(t)\right] \\
& \quad \in \mathrm{cl} \cdot \operatorname{co} \bigcup_{k=n}^{\infty} \mathscr{2}\left(t,\left(M x_{k}\right)(t)\right),
\end{aligned}
$$

so that, on letting $n \rightarrow \infty$,

$$
(v(t),(E x)(t)+(\Lambda x)(t)) \in 2(t,(M x)(t))
$$

by property (Q) (cf. Hou [12]). Obviously (4.8) is true for a.a. $\iota$ in $J$.

Now we show that there exists a measurable function $u: J \rightarrow U$ such that for a.e. $t$ in $J$ :

(i) $(E x)(t)+(\Lambda x)(t)=g(t, u(t))$,

(ii) $u(t) \in \omega(t)$ and

(iii) $v(t) \geqslant f_{0}(t,(M x)(t), u(t))$. To this end, define

$$
\mathcal{W}(t) \equiv\left\{u \in \omega(t): v(t) \geqslant f_{0}(t,(M x(t), u)\} .\right.
$$

We note first that each $\mathcal{W}(t)$ is closed in $U$ because $f_{0}$ is Carathéodory. We claim: $\mathcal{W}$ is a measurable multifunction from $J$ or $U$. Indeed, the map $f_{0}(t,(M x)(t), u)$ is measurable from $J \times U$ to $\mathbb{R}$ (cf. Appendix, theorem 3), hence so is the function $\mathscr{K}(t, u) \equiv v(t)-$ $f_{0}(t,(M x)(t), u)$. Then we have $G r(\mathcal{W})=G r(\omega) \cap \mathscr{K}^{-1}\left(\mathbb{R}^{+}\right)$, proving that $\mathcal{W}$ is measurable (cf. Hou [13], corollary 3.5); $\mathbb{R}^{+}$denoting the set of nonnegative real numbers.

In this manner, the problem of finding a $u$ satisfying (i), (ii) and (iii) is reduced to that of finding a measurable selection $u$ of $W$ such that $(E x)(t)+(\Lambda x)(t)=g(t, u(t))$ a.e. The existence of such a measurable selection follows by applying the implicit function theorem (cf. Appendix) in the same way as in the proof of theorem 3.2.

Now the pair $(x, u)$ is admissible and hence belongs to $\Omega$. It remains to show $I[x, u] \leqslant r$. By (L3) we have $f_{0}(t,(M x)(t), u(t)) \geqslant-\psi(t)+\langle b, g(t, u(t))\rangle$ and so $f_{0}(t,(M x)(t), u(t))$ is bounded below by an integrable function. As $f_{0}(t,(M x)(t), u(t)) \leqslant v(t)$, hence 
$f_{0}(t,(M x)(t), u(t))$ is integrable and

$$
I[x, u]=\int_{J} f_{0}(t,(M x)(t), u(t)) \mathrm{d} t \leqslant \int_{J} v(t) \mathrm{d} t \leqslant r,
$$

proving $I[x, u]=r$. This completes the proof.

\section{THE CASE IN WHICH $E=\mathrm{d} / \mathrm{d} t$}

We shall apply the results of section two, three and four to the case in which $E \equiv \mathrm{d} / \mathrm{d} t$, the derivative is in the sense of distribution. Thus $(\mathrm{P})$ becomes an evolution equation of the form

$$
\begin{aligned}
& \frac{\mathrm{d} x}{\mathrm{~d} t}(t)+(\Lambda x)(t)=g(t, u(t)), \quad x(0)=x_{0}, \\
& u(t) \in \omega(t) \quad \text { a.e. in } J .
\end{aligned}
$$

We give an interpretation of the initial condition $x(0)=x_{0}$.

Suppose assumptions (A2)-(A4) hold, that is, the operator $\left(N_{u} x\right)(t) \equiv(\Lambda x)(t)-$ $g(t, u(t))$ maps every $x$ in $X \equiv L_{p}(J, V)$ to an element $N_{u} x$ in $X^{*}$. Thus if $x$ satisfies (5.1) for a certain control $u$, then $x$ in $X$ and $\mathrm{d} x / \mathrm{d} t \in X^{*}$. In other words, $x$ is an element of the space

$$
W \equiv\left\{x \in X: \mathrm{d} x / \mathrm{d} t \in X^{*}\right\} .
$$

$W$ is a Banach space when endowed with the norm

$$
\|x\|_{W} \equiv\|x\|_{X}+\|\mathrm{d} x / \mathrm{d} t\|_{X^{*}}
$$

Denote by $C[J, H]$ the space of all continuous functions on $J$ with values in $h$. Then $W \subset$ $C[J, H]$ with continuous imbedding (cf. Carroll [7]). Since elements of $W$ are strictly speaking, not functions defined on $J$, but rather equivalence classes of such functions defined and equal up to sets of measure zero, we must clarify what is meant by the imbedding of $W$ into $C[J, H]$. What is intended is that the equivalence class $x \in W$ should contain an element belonging to the continuous function space that is the target of the imbedding and bounded in that space by a constant times $\|x\|_{W}$. More precisely, $W G C[J, H]$ means that for each $x \in W$, when considered as a function, can be redefined on a set of measure zero in the open interval $(0, T)$ in such a way that the modified function $x$ (which equals $x$ in $W$ ) is continuous on $(0, T)$ with values in $H$ and can be extended to a continuous function, again denoted by $x$, on $[0, T]$, and satisfies $\sup |x(t)|_{H} \leqslant C\|x\|_{W}$ where $C$ is a constant independent of $x$.

Thus if $x \in W$,

$$
\lim _{t \rightarrow 0+} x(t)
$$

exists, and we always interpret $x(0)=x_{0}$ to mean

$$
\lim _{t \rightarrow 0+} x(t)=x_{0} .
$$

Similarly we interpret

$$
x(T)=\lim _{t \rightarrow T_{-}} x(t)
$$

In the sequel, $\mathrm{d} x / \mathrm{d} t, x^{\prime}$ are used interchangeably to denote the distributional derivative of $x$. 
We consider first the initial condition $x(0)=x_{0}=0$.

LEMma 5.1. Define $E \equiv \mathrm{d} / \mathrm{d} t$, in the sense of distribution, from $X \equiv L_{p}(J, V)$ to $X^{*}$ with domain $D(E) \equiv\left\{x \in X: \mathrm{d} x / \mathrm{d} t \in X^{*}, x(0)=0\right\}$. Then $E$ is a linear, maximal monotone operator.

Proof. That $E$ is linear is evident. The monotonicity of $E$ follows from the inequality

$$
\begin{aligned}
\{E x, x\} & =\left\{x^{\prime}, x\right\}=\int_{J}\left\langle x^{\prime}(s), x(s)\right\rangle \mathrm{d} s \\
& =\frac{1}{2}\left[|x(T)|_{H}^{2}-|x(0)|_{H}^{2}\right]=\frac{1}{2}|x(T)|_{H}^{2} \geqslant 0 .
\end{aligned}
$$

Let $(y, z) \in X \times X^{*}$ and $\{z-E x, y-x\} \geqslant 0$ for all $x \in D(E)$. If we can show $y \in D(E)$ and $z=y^{\prime}$, then $E$ is maximal monotone. For this, let $x=\psi v$ where $v \in V$ and $\psi \in \mathscr{D}(J)$, the space of all infinitely differentiable real-valued functions with compact supports in the open interval $(0, T)$. Clearly $x \in D(E)$ and

$$
\begin{aligned}
0 & \leqslant\left\{z-\psi^{\prime} v, y-x\right\} \\
& =\{z, y\}-\left\{\psi^{\prime} v, y\right\}-\{z, \psi v\}+\left\langle\psi^{\prime} v, \psi v\right\} \\
& =\{z, y\}-\left\langle\int_{J}\left[\psi^{\prime}(s) y(s)-\psi(s) z(s)\right] \mathrm{d} s, v\right\rangle,
\end{aligned}
$$

the last term $\left\{\psi^{\prime} v, \psi v\right\}=0$ since $\psi$ has compact support. As $v$ being arbitrary, we have

$$
\int_{J} \psi^{\prime}(s) y(s) \mathrm{d} s=\int_{J} \psi(s) z(s) \mathrm{d} s \text { for all } \psi \in \mathscr{D}(J) .
$$

This implies $z=y^{\prime}$.

It remains to show $y(0)=0$. Let $x(t) \equiv t v / T$ for every $t \in J$ where $v \in V$. Obviously $x \in$ $D(E)$. Then

$$
\begin{aligned}
0 \leqslant\left\{y^{\prime}-x^{\prime}, y-x\right\} & =\int_{J}\left\langle y^{\prime}(s)-x^{\prime}(s), y(s)-x(s)\right\rangle \mathrm{d} s \\
& =\frac{1}{2}\left[|y(T)-x(T)|_{H}^{2}-|y(0)-x(0)|_{H}^{2}\right] \\
& =\frac{1}{2}\left[|y(T)-v|_{H}^{2}-|y(0)|_{H}^{2}\right] .
\end{aligned}
$$

Thus $|y(0)|_{H}^{2} \leqslant|y(T)-v|_{H}^{2}$. Since $V$ is dense in $H$ and $v$ is arbitrary in $V$, we assert that $y(0)=0$. This completes the proof.

Remark. In the proof of lemma 5.1, we have used the following formula. For any $x, y \in W$,

$$
(x(t), y(t))_{H}-(x(s), y(s))_{H}=\int_{s}^{t}\left\langle x^{\prime}(r), y(r)\right\rangle \mathrm{d} r+\int_{s}^{t}\left\langle y^{\prime}(r), x(r)\right\rangle \mathrm{d} r
$$

where $t, s \in J$ (cf. Carroll [7]).

In view of lemma 5.1 , all the results of sections $2-4$ are valid for $E=\mathrm{d} / \mathrm{d} t$ with $D(E)=$ $\left\{x \in X: E x \in X^{*}, x(0)=0\right\}$. However, we can obtain more interesting results about $\Phi$ defined in theorem 2.3. We need the definition below. 
Definition 5.2. Define $\Phi(t) \equiv\{x(t) \in H: x \in \Phi\}$ for $0<t \leqslant T$ and $\Phi(0) \equiv\{0\}$.

Thus $\Phi(t)$ is the attainable set of the system described by (5.1), (5.2) at time $t$. With abuse of notation, we also employ the same symbol $\Phi$ to denote the multifunction $t \rightarrow \Phi(t)$ from $J$ to $H$.

THEOREM 5.3. Suppose the hypotheses of theorem 3.2 are satisfied with $E=\mathrm{d} / \mathrm{d} t$ and $D(E)=\left\{x \in X: E x \in X^{*}\right.$ and $\left.x(0)=0\right\}$. Then the multifunction $\Phi$ is upper semicontinuous from $J$ to $H_{w}$ with weakly compact values, where $H_{w}$ is the space $H$ with weak topology.

Proof. If we are able to show that for every $t_{n} \rightarrow t_{0}, \xi_{n} \in \Phi\left(t_{n}\right)$, we can extract a subsequence of $\left\{\xi_{n}\right\}$ converging weakly to a point in $\Phi\left(t_{0}\right)$, then the assertion of theorem 5.3 follows from theorem 4.9 of Hou [12]. By definition of $\Phi(t)$, there are $x_{n} \in \Phi$ such that $x_{n}\left(t_{n}\right)=\xi_{n}$. Since $\Phi$ is sequentially compact (theorem 3.2), we can assume $x_{n} \rightarrow x \in \Phi$, and so it is enough to show $x_{n}\left(t_{n}\right) \rightarrow x\left(t_{0}\right)$. Let $v \in V$, we can write

$$
\left\langle v, x_{n}\left(t_{n}\right)-x\left(t_{0}\right)\right\rangle=\left\langle v, x_{n}\left(t_{n}\right)-x_{n}\left(t_{0}\right)\right\rangle+\left\langle v, x_{n}\left(t_{0}\right)-x\left(t_{0}\right)\right\rangle .
$$

For the first term on the right-hand side of (5.4),

$$
\begin{aligned}
\left|\left\langle v, x_{n}\left(t_{n}\right)-x_{n}\left(t_{0}\right)\right\rangle\right| & =\left|\int_{t_{n}}^{t_{0}}\left\langle v, x_{n}^{\prime}(s)\right\rangle \mathrm{d} s\right| \\
& \leqslant\|v\| \| x_{n}^{\prime}||_{X^{*}}\left|t_{n}-t_{0}\right| \rightarrow 0
\end{aligned}
$$

as $n \rightarrow \infty,\left\{x_{n}^{\prime}\right\}$ being bounded as $x_{n}^{\prime} \rightarrow x^{\prime}$. For the second term on the right-hand side of (5.4), we have

$$
\left\langle v, x_{n}\left(t_{0}\right)-x\left(t_{0}\right)\right\rangle=\int_{0}^{t_{0}}\left\langle v, x_{n}^{\prime}(s)-x^{\prime}(s)\right\rangle \mathrm{d} s=0
$$

as $x_{n}^{\prime} \rightarrow x^{\prime}$. Therefore we have proved that $\left\langle v, x_{n}\left(t_{n}\right)\right\rangle \rightarrow\left\langle v, x\left(t_{0}\right)\right\rangle$ for every $v \in V$. The fact that $V$ is dense in $H$ implies that $x_{n}\left(t_{n}\right) \rightarrow x\left(t_{0}\right)$ in $H$. This completes the proof.

Corollary 5.4. $\Phi(t)$ is weakly compact in $H$ for $0 \leqslant t \leqslant T$.

We are now able to state an existence theorem of time optimal control of intercepting a moving target.

THEOREM 5.5. Suppose the hypotheses of theorem 5.3 are satisfied. Let $t \rightarrow S(t)$ be a sequentially closed multifunction from $J$ to $H_{w}$ (cf. Hou [12]). If

$$
\mathscr{K} \equiv\{t \in J: S(t) \cap \Phi(t) \neq \varnothing\}
$$

is nonempty and if $k \equiv \inf \mathscr{K}$, then $S(k) \cap \Phi(k) \neq \varnothing$.

Proof. Let $t_{n} \in \mathscr{K}, t_{n} \rightarrow k$, then there exists $x_{n} \in \Phi$ such that $x_{n}\left(t_{n}\right) \in S\left(t_{n}\right) \cap \Phi\left(t_{n}\right)$. Since $\Phi$ is upper semicontinuous with compact valued from $J$ to $H_{w}$. We may assume that $x_{n}\left(t_{n}\right) \rightarrow x(k)$ with $x \in \Phi$ (cf. Hou [12, theorem 4.9]). Now we also have $x(k) \in S(k)$ since $S$ is sequentially closed. Hence $x(k) \in S(k) \cap \Phi(k)$, proving that $S(k) \cap \Phi(k) \neq \varnothing$. This completes the proof. 
We now treat below the case that $x(0)=x_{0} \neq 0$ but $x_{0} \in V$. If this is the case, we can always reduce back to the previous case $x(0)=0$ by a technical device.

Taking $w(t) \equiv x(t)-x_{0}$ and introducing $w$ instead of $x$ as our unknown function, we note that $w$ satisfies the initial value problem

$$
\begin{aligned}
& \frac{\mathrm{d} w}{\mathrm{~d} t}(t)+(B w)(t)=g(t, u(t)) \\
& w(0)=0, \\
& u(t) \in \omega(t) \quad \text { a.e. in } J .
\end{aligned}
$$

where the operator $B$, defined by $B w \in \Lambda\left(w+x_{0}\right)$, is still sequentially weakly continuous as $\Lambda$ is. If further $B$ satisfies (A4) in place of $\Lambda$, then all the theorems in sections 2-4 are valid for (5.5) and (5.6).

\section{THE PERIODIC CASE}

We define $E \equiv \mathrm{d} / \mathrm{d} t$, in the sense of distribution, as in section five but with a different domain $D(E) \equiv\left\{x \in X: E x \in X^{*}, x(0)=x(T)\right\}$. Thus the system (P) becomes

$$
\begin{aligned}
& \frac{\mathrm{d} x}{\mathrm{~d} t}(t)+(\Lambda x)(t)=g(t, u(t)), x(0)=x(T), \\
& u(t) \in \omega(t) \text { a.e. in } J .
\end{aligned}
$$

LEMMA 6.1. If $E$ is the operator $\mathrm{d} / \mathrm{d} t$ with domain $D(E)$, then $E$ is a linear, maximal monotone operator from $X$ to $X^{*}$.

Remark. In view of this lemma, all the results in sections two, three and four are applicable to $(6.1)$ and $(6.2)$.

Proof. That $E$ is linear is evident and the monotonicity of $E$ follows from

$$
\{E x, x\}=\int_{J}\left\langle x^{\prime}(s), x(s)\right\rangle \mathrm{d} s=\frac{1}{2}\left[|x(T)|_{H}^{2}-|x(0)|_{H}^{2}\right]=0 .
$$

To show $E$ is maximal monotone, let $(y, z) \in X \times X^{*}$ and $\{z-E x, y-x\} \geqslant 0$ for all $x \in$ $D(E)$. As in the proof of lemma 5.1, we may assert $z=y^{\prime}$. It remains to show $y(0)=y(T)$. For this, we note that for all $x \in D(E)$.

$$
\begin{aligned}
0 & \leqslant\left\langle y^{\prime}-x^{\prime}, y-x\right\rangle \\
& =\frac{1}{2}\left[|y(T)-x(T)|_{H}^{2}-|y(0)-x(0)|_{H}^{2}\right] \\
& =(x(0), y(0)-y(T))_{H}+\frac{1}{2}|y(T)|_{H}^{2}-\frac{1}{2}|y(0)|_{H}^{2} .
\end{aligned}
$$

The fact that $x(0)$ is arbitrary implies that $y(0)=y(T)$. This proves that $E$ is maximal monotone.

\section{THE CASE IN WHICH $E=\mathrm{d} / \mathrm{d} t$ AND $\Lambda$ IS LINEAR}

We consider in this section the control system (P) with $E=\mathrm{d} / \mathrm{d} t$ and $\Lambda$ determined by a bilinear form. This is mainly the kind of systems studied by Lions [18].

For each $t$ in $J$, we are given a continuous bilinear form $a(t ; v, w)$ on $V \times V$ to $\mathbb{R}$ having the 
following properties:

For all $v, w \in V$, the function $t \rightarrow(t ; v, w)$ is measurable and

$$
|a(t ; v, w)| \leqslant L\|v\|\|w\|
$$

where $L$ is a constant independent of $t, v$ and $w$. Also there exists $\alpha>0$ such that

$$
a(t ; v, v) \geqslant \alpha\|v\|^{2} \text {, for all } v \in V .
$$

Thus for fixed $v \in V$, the linear form $w \rightarrow a(t ; v, w)$ is continuous on $V$, therefore it can be written as

$$
a(t ; v, w)=\langle A(t) v, w\rangle, A(t) v \in V^{*} .
$$

We also deduce from (7.1) that

$$
\|A(t) v\| \leqslant L\|v\|, \text { for all } v \in V .
$$

Then $\{A(t)\}_{t \in J}$ is a family of continuous linear operators from $V$ to $V^{*}$, and because of (7.2), it is also coercive; i.e. for each $t$ of $J$,

$$
\langle A(t) v, v\rangle \geqslant \alpha\|v\|^{2}, \text { for all } v \in V .
$$

If we let $\Lambda$ be the operator from $X \equiv L_{2}(J, V)$ to $X^{*}=L_{2}\left(J, V^{*}\right)(p=2)$ defined by

$$
(\Lambda x)(t) \equiv A(t) x(t), \text { for every } t \in J, x \in X .
$$

Then $\Lambda$ is linear and continuous with norm less than $L$.

The system $(\mathrm{P})$ in which we are interested becomes

$$
\begin{aligned}
& \frac{\mathrm{d} x}{\mathrm{~d} t}(t)+(\Lambda x)(t)=g(t, u(t)), x(0)=x_{0} \in H, \\
& u(t) \in \omega(t) \quad \text { a.e. in } J .
\end{aligned}
$$

we observe first that if (7.7) has a solution, it is unique.

LEMMA 7.1. If $(x, u),(y, u)$ are two admissible pairs for (7.7), (7.8) with the same admissible control $u$, then $x=y$.

Proof. If $(x, u),(y, u)$ are two admissible pairs, then

$$
\left\langle x^{\prime}(t)-y^{\prime}(t), x(t)-y(t)\right\rangle+\langle(\Lambda x)(t)-(\Lambda y)(t), x(t)-y(t)\rangle=0 .
$$

and $x(0)=y(0)$. Now by $(7.5)$ we get

$$
\left\langle x^{\prime}(t)-y^{\prime}(t), x(t)-y(t)\right\rangle \leqslant-\alpha\|x(t)-y(t)\|^{2} \leqslant 0 .
$$

Integrating from 0 to $t$ and using (5.3), we obtain $\frac{1}{2}|x(t)-y(t)|_{H}^{2} \leqslant 0$, hence $x=y$. Lemma 7.1 is thereby proved.

A solution to (7.7) for a given $u$ is equal to $x=x_{1}+x_{2}$ where $x_{1}$ satisfies

$$
\frac{\mathrm{d} x}{\mathrm{~d} t}(t)+(\Lambda x)(t)=0, x(0)=x_{0} \in H
$$

This cquation is uncontrollable. $x_{2}$ is a solution to (7.7) but with initial condition $x_{2}(0)-0$. 
That (7.9) always has a unique solution follows from a theorem of Lions [18, theorem 1.2, p. 102]. Thus we may as well assume that the initial condition $x_{0}$ in (7.7) is always equal to 0 .

We demonstrate next that the results of section 2 are applicable here.

THEOREM 7.2. (existence and uniqueness of solutions). Suppose that $\Lambda$ satisfies (7.4), (7.5) and the map $g$ satisfies (B1) of section 3. Then (7.7) (with $x_{0}=0$ ) has a unique solution for a given measurable selection $u$ of $\omega$.

Proof. We need only to show that the hypotheses of theorem 2.3 , i.e. (A1)-(A4), are satisfied. (A1) holds since $E=\mathrm{d} / \mathrm{d} t$ with domain $D(E) \equiv\left\{x \in X: E x \in X^{*}, x(0)=0\right\}$ is linear and maximal monotone by lemma 5.1. Since $\Lambda$ is linear and continuous, (A2) clearly holds. (A3) is implied by (B1). $\Lambda$ s for $(\Lambda 4)$, we have to produce a bounded closed convex subset $K$ containing 0 in its interior such that $\left\{N_{u} x, x\right\} \geqslant 0$ for all $x \in \partial K . N_{u}$ is defined by $\left(N_{u} x\right)(t) \equiv(\Lambda x)(t)-g(t, u(t))$. We note that, by (7.5) and (B1),

$$
\begin{aligned}
\left\{N_{u} x, x\right\} & =\int_{J}\left\langle\left(N_{u} x\right)(t), x(t)\right\rangle \mathrm{d} t \\
& =\int_{J}\langle A(t) x(t), x(t)\rangle \mathrm{d} t-\int_{J}\langle g(t, u(t)), x(t)\rangle \mathrm{d} t \\
& \geqslant \int_{J} \alpha\|x(t)\|^{2} \mathrm{~d} t-\int_{J}\|g(t, u(t))\|_{*}\|x(t)\| \mathrm{d} t \\
& \geqslant\|x\|_{X}\left[\alpha\|x\|_{X}-\|l\|_{L_{2}(J, R)}\right] .
\end{aligned}
$$

Thus if we choose $K \equiv\left\{x \in X:\|x\|_{X} \leqslant C\right\}$, then, for sufficiently large $C$, we have $\left\{N_{u} x, x\right\} \geqslant 0$ for all $x \in \partial K$, establishing the validity of (A4).

Now it follows from theorem 2.3 that (7.7) with $x_{0}=0$ has a solution in $K$. Uniqueness of solution is immediate from lemma 7.1. Theorem 7.2 is thereby proved.

It is also clear that the results of sections 3 and 4 are applicable to the system (7.7), (7.8).

\section{AN EXAMPLE}

Let $G$ be an open bounded subset in the $n$-dimensional Euclidean space $\mathbb{R}^{n}$ with sufficiently smooth boundary. We denote points of $G$ by $\xi=\left(\xi_{1}, \ldots, \xi_{n}\right)$. We consider real-valued functions $x(\xi)$ defined on $G$ and belonging to the Sobolev space $W_{p}^{m}(G)$ (cf. Adams [1]) where $m$ is a natural number and $p>1$. Let $\alpha$ be a multi-index, $\alpha=\left(\alpha_{1}, \ldots, \alpha_{n}\right)$, where $\alpha_{i}$ are nonnegative integers. The Sobolev space is defined by

$$
W_{p}^{m}(G) \equiv\left\{x \in L_{p}(G, \mathbb{R}): D^{\alpha} x \in L_{p}(G, \mathbb{R}) \text { for all }|\alpha| \leqslant m\right\}
$$

with the norm given by

Here

$$
\|u\| \equiv\left(\sum_{|\alpha| \leqslant m} \int_{G}\left|\mathrm{D}^{\alpha} x\right|^{p} \mathrm{~d} \xi\right)^{1 / p}
$$

$$
\mathrm{D}^{\alpha} \equiv\left(\frac{\partial}{\partial \xi_{1}}\right)^{\alpha_{1}} \ldots\left(\frac{\partial}{\partial \xi_{n}}\right)^{\alpha_{n}}
$$

the derivatives are in the sense of distributions. 
Let $\mathscr{D}(G)$ be the family of infinitely differentiable functions with compact support in $G$. The closure of $\mathscr{D}(G)$ in the metric of $W_{p}^{m}(G)$ is denoted by $\mathscr{W}_{p}^{m}(G) . \mathscr{W}_{p}^{m}(G)$ is a separable reflexive Banach space for $1<p<+\infty$.

Let $n=3, p=2$ and $m=1$. Let $V \equiv \stackrel{\circ}{W}_{2}^{1}(G)$ and $H \equiv L_{2}(G, \mathbb{R})$. Then $V \subset H \subset V^{*}$.

We consider here a problem of heat transfer in an inhomogeneous bounded body $G \subset \mathbb{R}^{3}$ of sufficiently smooth boundary. This example is an extension of the case considered by Quilghini [19]. The system to be controlled is described by the equation

$$
\begin{aligned}
& \frac{\partial x(\xi, t)}{\partial t}=\operatorname{div}[k(\xi) \nabla x(\xi, t)]+q(t, u(\xi, t)) f(\xi) \\
& x(\xi, t)=0 \text { for all } \xi \in \partial G, t \in[0, T], \\
& x(\xi, 0) \equiv \lim _{t \rightarrow 0+} x(\xi, t)=0, \xi \in G .
\end{aligned}
$$

where $k_{1} \geqslant k(\xi) \geqslant k_{0}>0$ for all $\xi$ in $G$.

Here $x(\xi, t)$ describes the change of temperature at point $\xi$ at time $t$ and $q(t, u(\xi, t)) f(\xi)$ describes the amount of heat supplied by a source of heating output $q(t, u(\xi, t))$ which is distributed on $G$ proportionally to the values $f(\xi) \geqslant 0$ of a function $f \in H$.

Let $J \equiv[0, T]$. The function $q: J \times \mathbb{R} \rightarrow \mathbb{R}$ is a Carathéodory map; i.e. $q(\cdot, s)$ is measurable for fixed $s$ and $q(t, \cdot)$ is continuous for almost all $t$ in $J$. We assume also that

$$
|q(t, s)| \leqslant \beta(t) \quad \text { a.e. in } J,
$$

where $\beta \in L_{\infty}(J, \mathbb{R})$. Each control $u: G \times J \rightarrow \mathbb{R}$ is a function such that for each $t$ in $J$, $u(t) \equiv u(\cdot, t)$ is an element of the control set $\omega(t)$ defined by

$$
\omega(t) \equiv\{v \in H: 0 \leqslant v(\xi) \leqslant C \quad \text { a.e. in } G\}
$$

where $C>0$. Let $U \equiv H$, then $\omega: J \rightarrow U$ is a measurable multifunction with closed convex values.

If we set

$$
a(t ; v, w) \equiv \int_{G_{\mathrm{f}}} k(\xi) \nabla v \cdot \nabla w \mathrm{~d} \xi
$$

for all $v, w \in V$, then it is easy to check that (7.1) and (7.2) hold with $\alpha=k_{0} / C_{1}$ where $C_{1}$ is a constant determined by the Poincaré's inequality. In this manner, (8.1) is reduced to (7.7) with $g(t, u(t))(\xi) \equiv q(t, u(\xi, t)) f(\xi)$ and $x_{0}=0$. Clearly $g$ satisfies the assumption (B1) because of (8.2).

We now want to prove $g$ is a Carathéodory map from $J \times U \rightarrow V^{*}$.

LEMMA 8.1. $g: J \times U \rightarrow V^{*}$ is Carathéodory.

Proof. We prove first that $g(t, u)$ is demicontinuous in $u$. Let $\left\{u_{n}\right\}$ be a sequence in $U$ strongly convergent to $u$. Then we may assume $u_{n}(\xi) \rightarrow u(\xi)$ a.e. since $q$ is Carathéodory, we get $q\left(t, u_{n}(\xi)\right) \rightarrow q(t, u(\xi))$ a.e. Thus for any $v \in V$,

$$
\left\langle v, g\left(t, u_{n}\right)\right\rangle=\int_{G} v(\xi) q\left(t, u_{n}(\xi)\right) f(\xi) \mathrm{d} \xi \rightarrow \int_{G} v(\xi) q(t, u(\xi)) f(\xi) \mathrm{d} \xi=\langle v, g(t, u)\rangle .
$$

We conclude that $g$ is demicontinuous in $u$. 
To see that $g(\cdot, u)$ is strongly measurable from $J$ to $V^{*}$, it is enough to show $g(\cdot, u)$ is scalarly measurable since $V^{*}$ is separable (cf. Yosida [24]). To this end, let $v \in V^{* *}=V$. Then the function

$$
t \rightarrow\langle v, g(t, u)\rangle=\int_{G} v(\xi) q(t, u(\xi)) f(\xi) \mathrm{d} \xi
$$

is clearly measurable from $J$ to $\mathbb{R}$ by Fubini's theorem. this completes the proof of lemma 8.1 .

By theorem 7.2, (8.1) has a unique admissible trajectory for any given measurable selection $u$ of $\omega$. If we can show that assumption (B2) of section 3 holds, then the set $\Phi$ of all admissible trajectories is sequentially weakly compact.

LEMMA 8.2. For almost every $t$ of $J$, the set $g(t, \omega(t))$ is closed and convex.

Proof. For convexity, let $z_{1}, z_{2} \in g(t, \omega(t))$ for some $t \in J$ and let $z \equiv \lambda z_{1}+(1-\lambda) z_{2}$ for fixed $\lambda, 0 \leqslant \lambda \leqslant 1$. By definition of $g(t, \omega(t))$, there are $v_{i} \in \omega(t), i=1,2$, such that $z_{i}(\xi)=$ $q\left(t, v_{i}(\xi)\right) f(\xi)$ for a.a. $\xi \in G$. Let us set $y(\xi) \equiv \lambda q\left(t, v_{1}(\xi)\right)+(1-\lambda) q\left(t, v_{2}(\xi)\right)$ for all $\xi \in G$. Then $y(\xi) \in q(t, \mathscr{K})$ a.e. in $G$ because $q(t, \mathscr{K})$ is convex, where $\mathscr{K}$ is the closed interval [0,C]. By Hou $[13$, theorem 4.5.] we are able to find a measurable function $v: J \rightarrow \mathbb{R}$ with $0 \leqslant v(\xi) \leqslant C$ and satisfy $y(\xi)=q(t, v(\xi))$ a.e. Therefore $v \in \omega(t)$ and $z=y f \in g(t, \omega(t))$, proving that $g(t, \omega(t))$ is convex.

For closedness of $g(t, \omega(t))$, let us fix a $t$ in $J$ such that $|q(t, s)| \leqslant \beta(t)$ for all $s \in \mathbb{R}$. This is possible by (8.2). Let $\left\{z_{n}\right\}$ be a sequence in $g(t, \omega(t))$ with $z_{n} \rightarrow z$ strongly in $V^{*}$. We claim: $z \in g(t, \omega(t))$. There are $v_{n} \in \omega(t)$ such that $z_{n}(\xi)=q\left(t, v_{n}(\xi)\right) f(\xi)$. Since $\left|q\left(t, v_{n}(\xi)\right)\right| \leqslant$ $\beta(t)$, we may assume $q\left(t, v_{n}(\cdot)\right)$ converges weakly to some $w$ in $H$. It is clear that $z=w f$. Since $q(t, \mathscr{K})$ is closed and convex, and $q\left(t, v_{n}(\xi)\right) \in q(t, \mathscr{K})$ for a.a. $\xi \in G$, it follows easily from Cesari's closure theorem that $w(\xi) \in q(t, \mathscr{K})$ a.e. By theorem 4.5. in Hou [13] we are able to find a measurable function $v: J \rightarrow \mathbb{R}$ with $0 \leqslant v(\xi) \leqslant C$ a.e. and satisfying $w(\xi)=$ $q(t, v(\xi))$ a.e. therefore $z=w f \in g(t, \omega(t))$, proving that $g(t, \omega(t))$ is closed. Lemma 8.2 is thereby proved.

It follows now from theorem 3.2 that the set $\Phi$ of all admissible trajectories is sequentially weakly compact. In particular, by corollary $5.4 \Phi(\mathrm{T})$ is weakly compact in $H$.

We consider the optimization problem of minimizing the deviation of temperature of the body from a preassigned temperature distribution $h(\xi), \xi \in G$ at time $T$; i.e., to minimize the functional

$$
I[x(T)] \equiv \int_{G}|x(\xi, T)-\hbar(\xi)|^{2} \mathrm{~d} \xi
$$

over $\Phi(T)$. It is obvious that $I$ is weakly lower semicontinuous on $H$. Hence $I$ has an absolute minimum in $\Phi(T)$.

\section{APPENDIX}

The theorem below is a Banach space version of Cesari's closure theorem (cf. Cesari [9] and Hou [12, theorem 5.4]).

THEOREM 1. Let $G$ be a measure space, $X$ a topological space and $E$ be a Banach space. Let $f: G \times X \rightarrow E$ be a multifunction that satisfies sequential property $(\mathrm{Q})$ with respect to $x$ in $X$, i.e., the set inclusion

$$
\bigcap_{n=1} \operatorname{cl} \operatorname{co} \bigcup_{k=n} f\left(t, x_{k}\right) \subseteq f(t, x)
$$


holds for almost all $t$ in $G$ and for any sequence $x_{n}$ converging to $x$ in $X$. Let $\xi, \xi, n=1,2, \ldots$, be integrable functions in $L_{1}(G, E)$, and $z, z_{n}, n=1,2, \ldots$, functions from $G$ to $X$, satisfying the relation $\xi_{n}(t) \in f\left(t, z_{n}(t)\right)$ almost everywhere in $G$, and such that $\xi_{n} \rightarrow \xi$ weakly in $L_{1}(G, E), z_{n}(t) \rightarrow z(t)$ in $X$ for almost all $t$ in $G$ as $n \rightarrow \infty$. Then $\xi(t) \in f(t, z(t))$ almost everywhere in $G$.

The next theorem is a variant of Filippov's implicit function theorem (cf. Hou [13, theorem 4.6]).

THEOREM 2 . Let $T$ be a measure space with a complete, $\sigma$-finite nonnegative measure, $Z$ a Banach space with a countable family $S \subset Z^{*}$ separating points of $Z$, and $X$ be a separable complete metric space. Let $\Gamma: T \rightarrow X$ be a measurable multifunction with closed values, $f$ a function from $T \times X$ to $Z$ such that $f(\cdot, x)$ is strongly measurable for $x \in X$ and $f(t, \cdot)$ is demicontinuous for almost every $t$ in $T$, and let $g: T \rightarrow Z$ be a strongly measurable function satisfying the relation $g(t) \in f(t, \Gamma(t))$ almost everywhere in $T$. Then there exists a measurable selection $u: T \rightarrow X$ for $\Gamma$ satisfying $g(t)=f(t, u(t))$ almost everywhere in $T$.

The following theorem concerns the strong measurability of Banach space valued functions.

THEOREM 3. Let $T$ be a measure space and $X, Y$ be Banach spaces. Let $F: T \times X \rightarrow Y$ be a Carathéodory function, i.e., $f(\cdot, x)$ is strongly measurable for every $x$ in $X$ and $f(t, \cdot)$ is demicontinuous for almost all $t$ in $T$; and $s$ be a strongly measurable function from $T$ to $X$. Then the composite function $h: t \rightarrow f(t, s(t))$ is strongly measurable from $T$ to $X$.

Proof. Since $s$ is strongly measurable, there exists a sequence of simple functions $s_{n}: T \rightarrow X$ such that $s_{n}(t) \rightarrow s(t)$ strongly for almost all $t$ in $T$. Thus for every $n$, the composite function $h_{n}(t) \equiv f\left(t, s_{n}(t)\right)$ is strongly measurable. By Pettis' theorem (cf. Yosida [24]) there exist sets $E_{n} \subset T$ of measure zero such that $h_{n}\left(T \backslash E_{n}\right)$ is separable for every

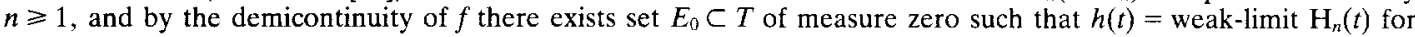
all $t$ in $T \backslash E_{0}$. Obviously the set

$$
E \equiv \bigcup_{n=0}^{\infty} E_{n}
$$

is of measure zero. The smallest closed linear subspace containing

$$
\bigcup_{n=1}^{\infty} h_{n}\left(T \backslash E_{n}\right)
$$

is clearly separable and contains $h(T \backslash E)$. Moreover, for every $x^{*}$ in $X^{*}$, the function $x^{*}(h)$ is measurable and $x^{*}\left(h_{n}(t)\right) \rightarrow x^{*}(h(t))$ for all $t$ in $T \backslash E$. Thus $h$ is essentially separably valued and scalarly measurable, whence strongly measurable by Pettis' theorem. This completes the pronf.

Acknowledgements - The author wishes to thank Professors L. Cesari and M. B. Suryanarayana for the stimulating conversations at the University of Michigan.

\section{REFERENCES}

1. Adams R. A., Sobolev Spaces, Academic Press, New York (1975).

2. AXElband E. I., Optimal control of linear distributed parameter systems, Advances in Control Systems (Edited by Leondes) vol. 7, pp. 258-310 Academic Press, New York (1969).

3. Aziz A. K., Wingate J. W. \& Bales M. J. (Editors), Control Theory of Systems governed by Partial Differential Equations, Academic Press, New York (1977).

4. BalAKRISHNAN A. V., Optimal control problems in Banach spaces, SIAM J. Control 3, 152-180 (1965).

5. BRÉZIS H., Equations et inequations nonlineaires dan les èspaces vectoriels en dualité, Annls Inst. Fourier Univ. Grenoble 18, 115-175 (1968).

6. BRÉZIS H., On some degenerate nonlinear parabolic equations, Nonlinear Functional Analysis, Proc. Symp. pure Math. Am. Math. Soc. 18, 28-38 (1970).

7. Carroll R. W., Abstract Methods in Partial Differential Equations, Harper \& Row (1969).

8. Cesari L., Convexity and property (Q) in optimal control theory, SIAM J. Control 12, 705-720 (1974).

9. Cesari L., Closure theorems for oriented fields and weak convergence, Archs ration. Mech. Anal. 55, 332-356 (1974).

10. Destel J., Remarks on weak compactness in $L_{1}(\mu, X)$, Glasg. Math. J. 18, part I, 87-91 (1977).

11. Dunfor N. \& Schwartz J., Linear Operators, Part I, Interscience, New York (1958).

12. Hou S. H., On property (Q) and other semicontinuity properties of multifunctions, Pacific J. Math. to appear.

13. Hou S. H., Implicit functions theorem in topological spaces, J. Applicable Analysis, to appear.

14. Hou S. H., Controllability and feedback systems, to appear.

15. Hille E. \& Phillips R. S., Functional Analysis and Semigroups, Am. math. Soc. Colloq. Publ. 31, Providence (1957). 
16. KenMOCHI N., Existence theorems for certain nonlinear equations, Hiroshima Math. J. 1, 435-443 (1971).

17. Leonard I. E., Smoothness and duality in $L_{p}(\mu, X), J$. math. Analysis Applic. 46, 513-522 (1974).

18. Lions J. L., Contrôle Optimal de Systèmes Governés par des Equations aux Derivées partielles, Dunod, Gauthier-Villars, Paris (1968).

19. QUiLghini D., Un esempio di controllo delle soluzioni dell' equation del calore, Rc. Semin. mat. Univ. Padova 36, 1-24 (1966).

20. Robinson A. C., A survey of optimal control of distributed parameter systems, Automatica 7, 371-388 (1971).

21. RuSSELL D. L., Controllability and stabilizability theory for linear partial differential equations: recent progress and open questions, SIAM Rev. 20, 639-739 (1978).

22. SERIERSTAD A., Comparness of sets of integrable functions with values in Banach spaces, proc. Am. math. Soc. 52, 204-208 (1975).

23. WARGa J., Optimal Control of Differential and Functional Equations, Academic Press, New York (1972).

24. Yosida K., Functional Analysis, Springer, New York (1965). 